\title{
Improving healthcare: a guide to roll-out best practices
}

\author{
Wilma ten Ham-Baloyi ${ }^{1}$, Karin Minnie ${ }^{2}$, Christa van der Walt ${ }^{3}$
}

1. Nelson Mandela University, Faculty of Health Sciences, Private Bag x 77000, Port Elizabeth, 6031, South Africa.

2. Director of INSINQ Focus Area, North-West University, Potchefstroom Campus, Private bag X6001, Potchefstroom, 2520, South Africa.

3. Honourable professor INSINQ Focus Area, North-West University, Potchefstroom Campus, Private bag X6001, Potchefstroom, 2520, South Africa.

\section{Author details:}

Karin Minnie(C.S.):Email: karin.minnie@nwu.ac.za, Tel: +27 (0) 18299 1836; Christa van der Walt (S.J.C.): Email: christa.vanderwalt2@gmail.com

\begin{abstract}
Background: Best practices comprise recent, relevant, and helpful nursing practices, methods, interventions, procedures or techniques based on high-quality evidence. Best practices should be implemented to improve individual patients' health outcomes the overall quality of health care, and to strengthen the health system at large. Four facilitators (benefit levers) to effectively roll-out best practices in healthcare organizations were identified: alignment, permeation plans, leadership for change, as well as supporting and reinforcing structures.

Purpose of the research: To develop and review a guide for an operational plan to facilitate the roll-out of best practices in healthcare organizations.

Methodology: The content of the guide was based on findings of an integrative literature review and interviews. This article discusses the development of the guide using the logic model as format (phase One) as well as its review including five key informants using the Delphi method (phase Two).

Results: The Logic Model provided a feasible format for the guide. Two Delphi rounds were required to review the guide's design and content. This guide addresses pre-roll-out resources; pre-roll-out activities, best practices (outcomes) during and after roll-outs; and impact.

Conclusion: The guide should be tested in different healthcare organizations and adapted accordingly to have an impact on improved patient and health outcomes. The guide can be used by managers wishing to roll-out best practices in their healthcare organizations to achieve improved health outcomes for individual patients.

Keywords: Best practices; guide for an operational plan; healthcare organizations; implementation of best practices; roll-out of best practices.

DOI: https://dx.doi.org/10.4314/ahs.v20i3.55

Cite as: Ten Ham-Baloyi W, Minnie K, van der Walt C. Improving healthcare: a guide to roll-out best practices. Afri Health Sci. 2020;20(3): 1487-1495. bttps://dx.doi.org/10.4314/abs.v20i3.55
\end{abstract}

\section{Introduction}

A best practice is more than practice based on evidence. It represents quality care which is deemed optimal. ${ }^{1}$

\author{
Corresponding author: \\ Wilma ten Ham-Baloyi, \\ Nelson Mandela University, \\ Faculty of Health Sciences, Private Bag x 77000, \\ Port Elizabeth, 6031, South Africa, \\ Tel: +27 (0) 41504 2959, \\ Fax: +27(0)41 5042960 , \\ Cell: $+27(0) 790745905$.
}

Email: wilma.tenham-baloyi@mandela.ac.za
Best practices are health practices, methods, interventions, procedures or techniques based on high-quality evidence in order to obtain improved patient and health outcomes. ${ }^{2}$ However, evidence alone is not sufficient to ensure evidence-based decision making but requires uptake and sustained implementation of the evidence. ${ }^{3}$ Globally evidence-based products such as best practice guidelines or standards are developed and made available, but the challenge remains to get evidence implemented and then rolled-out into everyday practice and clinical decision making. ${ }^{4}$ Numerous barriers to the implementation and roll-out of best practices have been reported, such as the complexity of practice environments, the lack of support of management and buy- 
in, resistance to change, and the suitability of the best practice for an individual patient. ${ }^{5}$ Furthermore, tailoring best practices to the needs and preferences of diverse populations requires further research and testing before rolling out and scaling up the best practice in a specific institution. ${ }^{6}$

Rolling-out or "spreading", involves the active disseminating of best practice and knowledge about an intervention and implementation of this intervention in every relevant available care setting in order to provide more patients with evidence-informed care. ${ }^{7,8}$ Roll-out of best practices further requires a point when evidence is accepted by most individuals and cannot be turned back and therefore change is inevitable: the so-called tipping point. ${ }^{9}$ To create that tipping point towards successful adoption, implementation and roll-out of evidence and to ensure that innovations (such as best practices) in the health system are rolled-out, certain facilitators are required.

The "Evidence Informed Model of Care" which was developed by Edwards and Grinspun, ${ }^{8}$ is supported by evidence and identifies the following four facilitators for roll-out (the so-called benefit levers): alignment, permeation plans (plans for roll-out), leadership for change and reinforcing and supporting structures. Alignment implies alignment among stakeholders (personal alignment), between top management, middle management and government (organizational alignment), and with the external environment, financial incentives, regulations and public reporting professional norms (contextual alignment). Permeation plans include three phases: 1) preparing the roll-out of best practices; 2) developing the plan; and 3) executing the plan. Leadership for change includes characteristics such as leadership types, leadership strategies, the position of leadership, the attitude of the leader and support for the leader in the organization. Supporting and reinforcing structures include various requirements such as: resources, time, education, communication, feedback and evaluation about the best practice. ${ }^{8,10}$ According to the model, roll-out of best practices occurs at certain levels in organizations and systems, such as individual level (the health professional in the unit of the organization), management level (the unit and the healthcare organizations at large), provincial level (such as the provincial Departments of Health) and national level (such as the National Departments of Health). ${ }^{8}$

Globally, and in South Africa, the roll-out of best practices remains challenging as developed best practices might remain inaccessible and unidentified for use. ${ }^{2,8}$ This, coupled with contextual factors creating barriers for implementation and roll-out, results in the best practice not being implemented and rolled-out and therefore practice is not changed. ${ }^{6}$ A guide, where these factors in the form of benefit levers are considered, could help organizations or departments to develop an operational plan that facilitates the roll-out of best practices in a certain context. An operational plan can be regarded as part of a strategic plan specifying how to operate in practice to implement actions and monitor plans. It will also define what the organisation's human, financial and other capacity requirements are and how to engage these resources. ${ }^{11}$ This operational plan could enhance the roll-out of best practices to improve patients' and health outcomes. Using a search on Google and GoogleScholar, no published guide for an operational plan could be found that facilitates the roll-out of best practices in South Africa.

\section{Aim}

This study aimed to develop and review a guide for an operational plan that facilitates the roll-out of best practices in a healthcare organization.

\section{Methods}

The content of the guide is based on the findings of two methods. Firstly, an integrative literature review regarding the benefit levers' characteristics for system-wide roll-out of best healthcare practices was conducted. ${ }^{10}$ A comprehensive search using multiple sites such as Scopus, EBSCOhost, ProQuest, ScienceDirect, Cochrane Library, Nexus, SAePublications, Sabinet, Google Scholar and grey literature was conducted (January-March 2012) and updated (December 2014). After a thorough search and selection process, 40 documents met the inclusion criteria. Thirty-five documents remained after critical appraisal. Data revealed characteristics about alignment (personal, organizational and contextual attributes), permeation plans (three phases: 1. Preparing for spread; 2. Developing the plan; and 3. Executing the plan), leadership for change (types of leaders, strategies for spread of best practices used by leaders, position of the leader in the organisation, attitude of the leader and support from the leader and to the leader) and supporting and reinforcing structures (types, including organisational structure and culture, contextual structure, individuals as structure and the innovation itself as a structure as well as requirements, including resources, time, education and development, communication, networking and cooperation as well as feedback and evaluation). ${ }^{10}$ Secondly, twelve indi- 
vidual semi-structured interviews were conducted with healthcare professionals' from various South African healthcare levels about their perspectives concerning the requirements for facilitating the roll-out of kangaroo mother care as a best practice. Content analysis were guided in terms of the four requirements for roll-out of best practices, identified in Edwards and Grinspun's Evidence Informed Model of Care. ${ }^{8,12}$ The requirements for the successful rollout of best practices mentioned by the participants in this study concur with the requirements of Edwards and Grinspun: personal alignment and protocol/policy alignment with the best practice; a roll-out plan; leadership; and supporting and reinforcing structures such as: resources, communicating, education and development regarding the best practice, and the organisational structure. The requirements were identified at four different levels: individual level (e.g. the nurse and medical specialists), management level (of the hospital), provincial level and national level. ${ }^{12}$ This article discusses the development of the guide using the Logic Model as format (phase 1) as well as its review using a Delphi method (phase 2). Both phases were conducted by the first author under supervision of the second and third authors.

\section{Developing the draft guide using a Logic Model (phase 1)}

A Logic Model is a systematic and visual way of presenting and sharing an understanding of the relationships among the resources required to operate a certain program, the activities planned, and the changes or results expected to achieve the change. ${ }^{13}$ Logic Models can therefore be used for program planning and design, such as a program to implement and roll-out a best practice. The Logic Model has four steps: Step 1: Preparation for the development of the Logic Model; Step 2: Development and assembling information; Step 3: Creation of the Logic Model; and Step 4: Reviewing and revising the logic model. ${ }^{14} \mathrm{~A}$ description and application of these steps in order to develop the guide is outlined in Table 1.

Table 1: The steps of the Logic Model applied to the guide ${ }^{13,14}$

\begin{tabular}{|c|c|}
\hline Steps of the Logic Model & Application of the steps for the guide \\
\hline $\begin{array}{l}\text { Step 1: Preparation for the development of } \\
\text { the Logic Model. } \\
\text { Consider: } \\
\begin{array}{l}\text { The type of stakeholders who should be } \\
\text { involved (such as program staff, board } \\
\text { members, funders) } \\
\text { - Decision making (and who has decision } \\
\text { making power) } \\
\text { - Resources } \\
\text { - The scope of the program/logic model }\end{array}\end{array}$ & $\begin{array}{l}\text { Based on the findings of an integrative literature review and } \\
\text { interviews with stakeholders involved in the implementation } \\
\text { and roll-out of a best practice: } \\
\text { - Stake-holders and decision making: leaders (who are } \\
\text { decision makers) from all levels of the health system } \\
\text { should be involved in the development of the } \\
\text { operational plan } \\
\text { Resources: Pre-implementation/roll-out of best } \\
\text { practices: existing resources and structures should be } \\
\text { analysed } \\
\text { Scope: developing a guide which can help to create an } \\
\text { operational plan for the use of benefit levers in the roll- } \\
\text { out of best practices in organizations and departments }\end{array}$ \\
\hline $\begin{array}{l}\text { Step 2: Development and assembling } \\
\text { information } \\
\text { During assembling and developing the } \\
\text { following should be considered: } \\
\text { - Resources } \\
\text { - Activities } \\
\text { - Outputs } \\
\text { - Outcomes } \\
\text { Outcomes can involve individual, community, } \\
\text { systematic, organizational, and short-term, } \\
\text { mid-term, and long-term outcomes }\end{array}$ & $\begin{array}{l}\text { - Resources/inputs } \\
\text { - Activities (pre-, during, and post roll-out) } \\
\text { - Outputs } \\
\text { - Impact } \\
\text { Findings identified per benefit lever were included, as well as } \\
\text { the levels responsible for carrying out the activities }\end{array}$ \\
\hline $\begin{array}{l}\text { Step 3: Creation of the Logic Model } \\
\text { The determination of the scope of logic model: } \\
\text { - What should be included and described } \\
\text { (content) and the depth (scope) }\end{array}$ & $\begin{array}{l}\text { - Content and scope: the interviews determined the scope } \\
\text { and content of the guide such as the specific levels and } \\
\text { activities pre-, during and post-implementation and roll- } \\
\text { out of best practices, using Edwards } \\
\text { and Grinspun's benefit levers as a framework } \\
\text { Layout: Word-document with a model outlining a } \\
\text { summary of the guide using rectangles } \\
\text { connected by arrows }\end{array}$ \\
\hline $\begin{array}{l}\text { Step 4: Reviewing and revising } \\
\text { the Logic Model } \\
\text { The Logic Model should be reviewed to } \\
\text { determine whether all relevant aspects such as } \\
\text { short and long-term } \\
\text { outcomes and objectives have been included; } \\
\text { whether the model is logical, and; } \\
\text { whether the layout is appropriate }\end{array}$ & $\begin{array}{l}\text { The draft guide was reviewed using an expert panel } \\
\text { (see phase 2) } \\
\text { Revision of the guide should be done at least annually }\end{array}$ \\
\hline
\end{tabular}


Reviewing the guide using the Delphi method (phase 2) The draft guide was reviewed and evaluated by key informants, using the Delphi method. Delphi is a commonly acknowledged method which measures the judgements of a group of experts for the purpose of decision making, often referred to as a consensus method, as it arrives at consensus usually through a few rounds. ${ }^{15,16,17}$

\section{Population and sampling}

The population of interest included key informants who were involved in the roll-out of best practices, fluent in English, and computer literate as e-mail was used to collect the data. The key informants were selected to provide their opinions on the design and content of the developed guide. The key informants were sampled using purposive sampling and recruited by email. In total 10 e-mails were sent to invite key informants from various institutions and levels of South Africa's health system to participate. Information regarding the study was sent to the key informants and consent was asked using email. Five $(n=5)$ key informants $(K)$ consented to participate, of whom two $(n=2)$ were from hospital level $(\mathrm{K} 1, \mathrm{~K} 2)$, two $(\mathrm{n}=2)$ from provincial level $(\mathrm{K} 3, \mathrm{~K} 4)$, and one $(n=1)$ from academic/researcher level (K5).

\section{Data-collection}

The guide and questionnaire were reviewed by the second and third authors prior to the data collection. The Delphi cycle of review was conducted using e-mail correspondence during two rounds.

In the first round, the draft guide, together with a brief explanation of the Logic Model and how this model was applied to develop the guide as well as a questionnaire were sent to the five key informants. The questionnaire contained both closed and open-ended questions. Items of the questionnaire included questions regarding four aspects: layout, formulation (design) and the feasibility and relevance (the content) of the draft guide. Each aspect included a 5-point Likert scale question ranking from "very bad" to "excellent" and one open-ended question requesting suggestions to improve the aspect of the draft guide. After completing the questionnaire, the key informants were asked to send their feedback via e-mail within a two-week timeframe.

The second round's email to the five key informants summarised the feedback of round 1 and presented the refined guide as well as the model that summarised the information in the guide enabling the key informants to reach a final consensus regarding the design and content of both the guide and the model. No questionnaire was required in this round but key informants were asked to provide final feedback again within a two-week timeframe. Consensus was reached after the second round, and no further rounds were necessary.

\section{Data-analysis}

Data from both rounds were analysed using descriptive analysis by providing simple summaries about the sample and the information that had been obtained, grouping similar items together. ${ }^{16}$ As the focus was on the development of the guide in terms of design (layout and formulation) and content (feasibility and contextual relevance), the content validity index did not need to be calculated.

\section{Ethical considerations}

The study obtained ethical permission from the NorthWest University (ethics number: NWU-00005-12-A1). Participation in the study was voluntary. Consent to participate was obtained per e-mail. Confidentiality was maintained as individual emails were sent per key informant anno names were asked in the questionnaires nor linked to the feedback of specific key informants.

\section{Trustworthiness}

To ensure rigour, the researcher kept an audit trail during the data collection using the Delphi method. ${ }^{17}$ High response rates were achieved by sending follow-up e-mails to non-respondents. Key informants were used who did not have conflicts of interest and had sufficient knowledge about and interest (experts) in the topic. ${ }^{16}$

\section{Results}

\section{Feedback from Delphi rounds}

A summary of the feedback of both rounds are indicated in Table 2. 
Table 2: Summary of the feedback of the key informants per round

\begin{tabular}{|l|l|}
\hline \multicolumn{2}{|l|}{ Round 1 } \\
\hline Aspect & Feedback \\
\hline Lay out & $\begin{array}{l}\text { "Average" (K3; K4) and "Very good" (K2) } \\
\text { All key informants indicated that the draft guide was too dense (K1; K2 and } \\
\text { K3). It was suggested to use one page per aspect of the guide (K1). Another } \\
\text { suggestion was made to attach a logic model graph in addition to the draft } \\
\text { guide aiming to clarify the aspects in the guide (K1). }\end{array}$ \\
\hline Formulation & $\begin{array}{l}\text { "Good" (K2; K3 and K4) } \\
\text { Editing was suggested (K1; K4), and removing of brackets which restricted } \\
\text { the flow of the content of the draft guide (K3). }\end{array}$ \\
\hline Feasibility & $\begin{array}{l}\text { "Feasible" (K2; K3) and "Average" (K4). } \\
\text { Recommendations regarding the draft guide's detail and applicability were } \\
\text { provided. For example, the guide should indicate more detail about its } \\
\text { operationalization (K1, K5), including practical examples as to how to } \\
\text { operationalise the guide (K4). The testability of the guide should be } \\
\text { specified, once it has been implemented in practice (K4). }\end{array}$ \\
\hline Relevance & $\begin{array}{l}\text { "Very relevant" (K2; K3) and "Relevant" (K4). } \\
\text { Comments included that support should be acquired for the roll-out of the } \\
\text { best practice at national level (K2; K3). }\end{array}$ \\
\hline by using arrows (K1).
\end{tabular}

\section{The reviewed guide}

The reviewed guide consisted of five boxes in which the different aspects according to the Logic Model are explained: resources/inputs; activities; outputs; outcomes and impact that are applicable pre, during and post rollout of best practices. The benefit levers (alignment, permeation plans, leadership for change and supporting and reinforcing structures) and the different levels of the health system (individual level, management level, provincial level and national level), were included in the relevant.

Figure 1 outlines the model that summarised the guide A more detailed content of each aspect of the guide will be discussed in the following sections: 


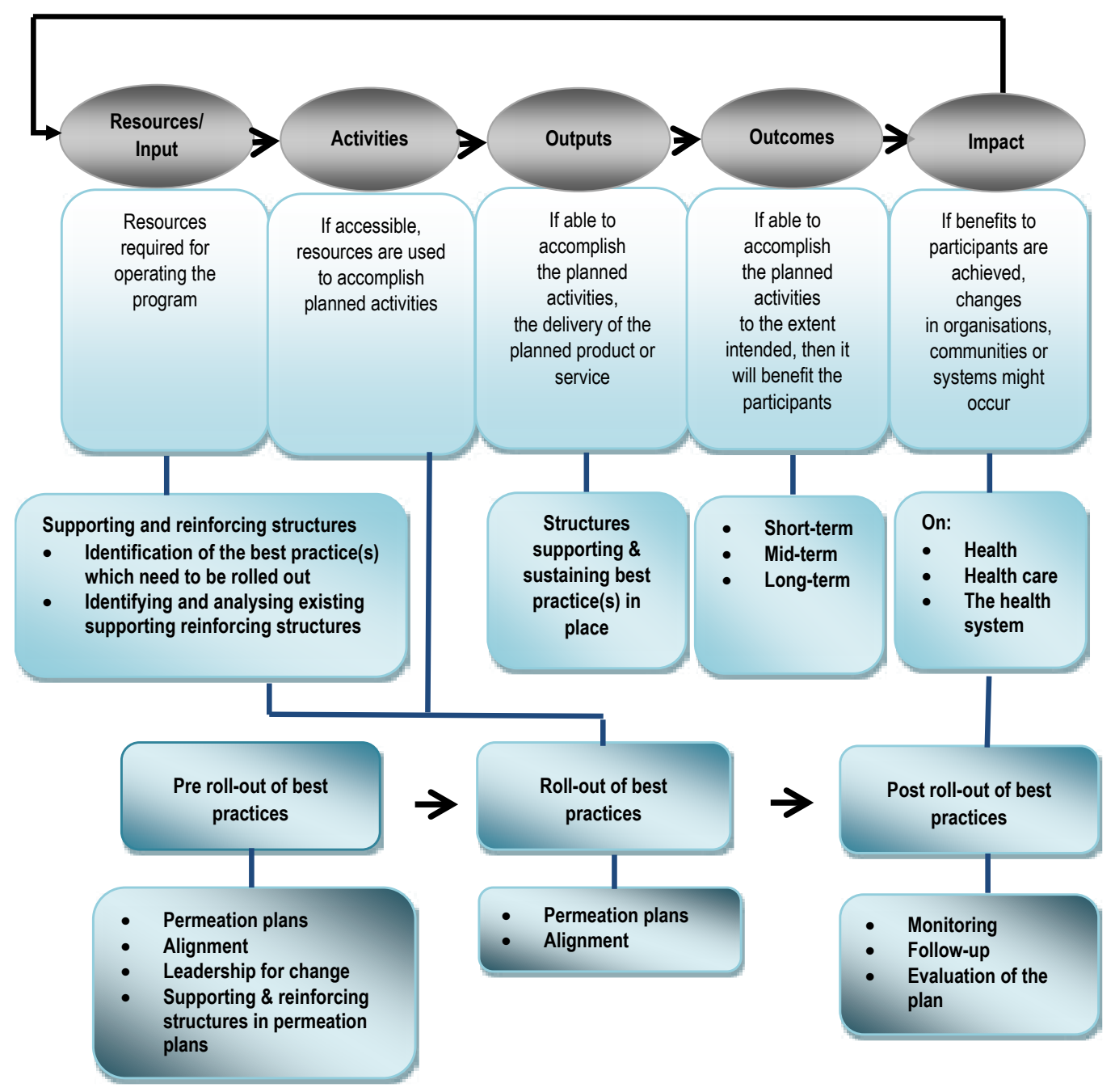

Figure 1: Model outlining the summary of the guide

\section{Resources/inputs (pre roll-out of best practices)}

Firstly, the best practice(s) that require roll-out should be identified by an organization, or country. Secondly, an analysis should be done about the existing and required resources. These include internal budget, external funding/sponsorship, equipment, human resources, infrastructure and implementation programmes and policies. For small-scale roll-out within an organisation these activities have to be executed by managers in the organization. For larger-scale roll-out, officials of district and provincial levels or sections of the Department of Health should reviewed by officials at national level with authority.

\section{Activities (pre roll-out of best practices)}

For small-scale roll-out of the best practice(s) within an organisation, a plan for roll-out should be developed at higher management level in collaboration with lower management levels. For larger scale roll-out, the order to develop a plan for roll-out (benefit level permeation plan) should be developed, executed and evaluated by officials at provincial level. The developed plan should receive buy-in by stakeholders at all levels of the health system or health organization. This was perceived as crucial as resistance towards the best practice(s) could exist at all levels.

Further, leadership at management and individual level was identified as crucial to the roll-out of the best practice(s). The tasks and roles of these leaders were also identified in the guide, such as providing technical and mental support, ensuring the continuity of the best practice, having a motivating/convincing role and raising enthusiasm, acting as a consultant and targeting the right people in an organization to successfully roll-out the best practice(s).

The guide further outlines the supporting and reinforcing structures in terms of responsibilities and activities at each level. At individual level knowledge should be shared, and meetings convened to evaluate the imple- 
mentation of the best practice(s). Orientation of new staff members regarding the best practice(s), having experienced/senior clinicians who are familiar with the best practice(s) are also important to facilitate the roll out of the best practice(s) which must be clearly and simply defined. The benefits should be clear to convince stakeholders to buy-in to the roll-out of the best practice(s). The management level within the organization should provide the following support structures: good infrastructure, human resources in terms of appointing dedicated and skilled staff members, equipment, internal budget and additional external funding, policies and mental support such as recognition and awards for personnel implementing good practices. When the rollout is done at a larger scale, management at provincial level should provide support structures: provincial policies, guidelines, documents and forms supporting the best practice(s), approval of a budget for infrastructure, existing implementation programs, sponsorships for training and congresses related to the best practice(s) and an information packages for hospitals regarding the best practice(s). Furthermore, a district clinical specialist team and a primary health team should be appointed, and coordination of training should be done to enhance communication and skills among staff and management. National level should provide infrastructure, strategies or a national policy/guideline that supports the roll-out of the best practice, and a national implementation task team to ensure national roll-out of the best practice(s).

\section{Activities (during roll-out of best practices)}

Under activities, the plan for roll-out should be executed. For small-scale roll-out, the officials at management level of an organization and for larger scale roll-out those at provincial level have to ensure that the roll-out plan is executed and feedback provided to the authorities at the relevant management levels. For both smalland larger-scale roll-outs, the stakeholders at individual level should execute the actual plan for roll-out. During the process of roll-out, the plan should be aligned with the existing supporting and reinforcing structures and the buy-in of all stakeholders sustained.

\section{Activities (post roll-out of best practices)}

Monitoring and follow-up of the roll-out of the best practice(s) should be done at management level (for small-scale roll-outs) or at provincial level and national level (for larger-scale roll-outs).

\section{Outputs (post roll-out of best practices)}

The outputs, in terms of all the (developed) structures supporting and sustaining the best practice(s) and the roll-out process, such as policies, acts, and resources should be in place at all the levels of the organization or health system.

\section{Outcomes (post roll-out of best practices)}

Outcomes include short-term outcomes such as improved skills, understanding, knowledge and intentions of the target group towards the take up, implementation and roll-out of the best practice(s). Mid-term outcomes could include improved behaviour and attitude towards the take up, implementation and roll-out of the best practice(s). Long-term outcomes include aspects such as enhanced uptake, implementation and roll-out of the best practice(s) as well as enabling more patients to receive high-quality care. The outcomes should be evaluated and updated regularly (for example annually) at the level of implementation.

\section{Impacts (post roll-out of best practices)}

The impacts of the roll-out of the best practice(s) which could be expected include improved health outcomes for individual patients, improved quality of care and evidence of organizational or health system's strengthening. The impacts should be evaluated over a term of 1,5 or 10 years to view the short-term versus the longterm impact of the roll-out of the best practice(s).

\section{Discussion}

This study was unique as no guide for an operational plan had been previously developed in South Africa and using the Logic Model format was also new. The guide is unique because the findings were identified per benefit lever at the levels responsible for carrying out specific activities in relation to the "Evidence Informed Model of Care".

This study acknowledges that roll-out of best practices can be done at a larger scale at different levels in the health system as well as at a smaller scale within organizations. For standardisation of nursing practice and the positive impact a best practice can make on patient-, nursing- and health outcomes, roll-out of best practices at a larger scale is recommended. ${ }^{8}$ This developed guide included the benefit levers because they were useful in the roll-out of best practices at small and large scales. The importance of the benefit levers and their role in the roll-out of best practices was confirmed by literature reports. The importance for the roll-out of 
best practices to get buy-in from all stakeholders at all levels has been supported by the Agency for Healthcare Research and Quality. ${ }^{18}$ Leadership is required at all levels of the health system to roll-out best practices. ${ }^{8}$ Different studies confirmed that the required leadership characteristics include the ability of the leader to teach, good communication skills, be well-trained, and being passionate or driven about changing practice. ${ }^{8}$ The importance of the motivating, convincing and advisory role of the leader was also mentioned by other researchers. ${ }^{8}$ Supporting and reinforcing structures such as resources, including policies, infrastructure and staff was found to be crucial by Edwards and Grinspun's study in order to roll-out best practices. ${ }^{8}$ This benefit lever was therefore included in the guide.

Levels were considered in the guide as this guide was developed and reviewed based on the South African context, which is in line with the South African health system. However, for adaptation the guide should be tested in different contexts by means of intervention research. Finally, as complexity of health systems could pose barriers for the roll-out of best practices, ${ }^{6}$ a better understanding of the influence of a system on the rollout of best practices, and the system's requirements for enhancing the roll-out of best practices are required for the effective sustained roll-out of best practice(s).

The guide can be used and adapted by managers in healthcare organizations and departments wishing to implement and roll-out best practices. Managers who wish to roll-out best practices in their healthcare organizations, should plan considerable time for the preparation of the roll-out, including conducting a context analysis of what is required to roll-out the best practice(s) in the specific organization. Evaluating and documenting the costs and cost-effectiveness of the best practice(s) should also be done to budget for rolling-out the best practice(s).

The guide for an operational plan was developed by the researcher and not a team. Although the guide was evaluated, additional insights might have been produced if more individuals participated during its development. The guide was informed by global literature, but key-informants of the interviews and the participants that reviewed the guide were South Africans. Therefore, the guide might require adaptation to be applicable to other contexts. The guide could be considered to be a draft as it has not yet been validated and tested. Further adjustments might be required after validation and piloting.

\section{Conclusion}

A Logic Model was found to be a feasible format for the development for a guide for an operational plan, as well as the inclusion of benefit levers at different levels of the health system. The guide could be used by managers, organizations and departments to develop an operational plan. The guide requires further adaptation and testing before it can be used in other contexts. Once contextualized, the guide may have an impact on improved health outcomes for individual patients, improved overall quality of healthcare, as well as the strengthening of the health system at large.

\section{Acknowledgements}

We are grateful to all the key informants for their participation and willingness to share their experiences and thoughts. This study did not receive funding.

\section{Conflict of interest}

The authors declare that they have no financial or personal competing interests.

\section{References}

1. Nelson AM. Best practice in nursing: a concept analysis. International Journal of Nursing Studies 2014; 51 (11): 1507-1516.

2. Makic MBF, Martin SA, Burns S, Philbrick D, Rauen C. Putting evidence into nursing practice: four traditional practices not supported by the evidence. Critical Care Nurse 2013; 33 (2): 28-44.

3. Straus S, Tetroe J, Graham ID. Knowledge Translation in Health Care: Moving from Evidence to Practice. $2^{\text {nd }}$ Ed. John Wiley \& Son: West Sussex, UK; 2013.

4. Madjid S, Foo S, Luyt B, Zhang XY-L, Theng Y-K, \& Chang Mokhtar IA. Adopting evidence-based practice in clinical decision making: nurses' perceptions, knowledge, and barriers. Journal of Medical Library Association 2011; 99 (3): 229-236.

5. Fitzgerald S, Geaney F, Kelly C, McHugh S, Perry IJ. Barriers to and facilitators of implementing complex workplace dietary interventions: process evaluation results of a cluster controlled trial. BMC Health Services Research 2016; 16 (139): 1-13.

6. Gitlin, LN. Introducing a New Intervention: An Overview of Research Phases and Common Challenges. American Journal of Occupational Therapy 2013; 67 (2): 177-184.

7. Ilott I, Gerrish K, Pownall S, Eltringham S, Booth A. Exploring scale-up, spread, and sustainability: an instrumental case study tracing an innovation 
to enhance dysphagia care. Implementation Science 2013; 29(8):128.

8. Edwards N, Grinspun D. Understanding whole systems change in healthcare: the case of emerging Evidence-informed Nursing service delivery models. Canadian Health Services Research Foundation: Ottowa; 2011.

9. Turner S, Morris S, Sheringham J, Hudson E, Fulop NJ. Study protocol: DEcisions in health Care to Introduce or Diffuse innovations using Evidence (DECIDE). Implementation Science 2016; 11 (48): 412-418.

10. Ten Ham W, Minnie K, \& van der Walt C. Integrative review of benefit levers' characteristics for system-wide spread of best healthcare practices. Journal of Advanced Nursing 2016; 72 (1): 33-49.

11. Beale W, Maquet M, Tua J. Resources for implementing the WWF Project and Programme Standards. www.panda.org/standards/2_3_operational_plan; 2007.

12. Ten Ham W, Minnie CS, van der Walt SJC. Health care professionals' perspectives on the requirements facilitating the roll-out of kangaroo mother care in South Africa. Health SA Gesondheid 2016; 21 (2016): 228-237

13. Innovation Network. Logic Model Workbook. www.innonet.org/client_docs/ File/logic_model_ workbook.pdf; 2016.

14. The Health Communication Unit. Logic Model Workbook. www.thcu.ca/infoandresources/.../logicmodel.wkbk.v6.1.full.aug; 2011.

15. Hsu CC, Sandford, BA. The Delphi-technique: making sense of consensus. http://pareonline.net/ pdf/v12n10.pdf; 2007.

16. Gray JR, Burns N, Grove SK. The practice of Nursing Research: Conduct, Critique and Utilization. 8th ed. Elsevier Saunders: St Louis; 2016.

17. Skulmoski GJ, Hartman FT, Krahn J. The Delphi Method for Graduate Research. http://www.jite. org/documents/Vol6/JITEv6p001-021Skulmoski212. pdf; 2007.

18. Agency for Healthcare Research and Quality. Preventing Falls in Hospitals How do you implement the fall prevention program in your organization? http://www.ahrq.gov/professionals/systems/hospital/fallpxtoolkit/fallpxtk4.html; 2013. 